$\xi=$

\title{
Numerical Study on Noise Reduction of Gas Extinguishing Nozzle Using Adsorbent
}

\author{
Hak-Sun Kim ${ }^{1}$, Yo-Hwan Kim ${ }^{1}$, In-Ju Hwang ${ }^{2}$ and Youn-Jea Kim ${ }^{3 *}$ \\ ${ }^{1}$ Graduate School of Mechanical Engineering, Sungkyunkwan University, Suwon 16419, Republic of Korea \\ ${ }^{2}$ Department of Future Technology and Convergence Research, Korea Institute of Civil Engineering and Building Technology, Goyang \\ 10223, Republic of Korea \\ ${ }^{3}$ School of Mechanical Engineering, Sungkyunkwan University, Suwon 16419, Republic of Korea \\ *Corresponding author E-mail: yjkim@skku.edu
}

\begin{abstract}
Currently, gas-based firefighting systems tend to release fire extinguishing agent at pressures of around 100 bar or more for quick firefighting. The flow noise produced by the agent discharge is approximately $140 \mathrm{~dB}$, which is similar to fireworks. This powerful sound pressure level (SPL) results in equipment failure during the fire suppression process, accelerating the movement studies to reduce flow noise. Most previous studies have selected design parameters such as packing density, porosity, and absorbent arrays, but these factors alone do not address noise reduction issues. The methods used in this study constitute the thickness of the absorber in the nozzle. The sound absorber plays an important role in absorbing sound pressure by flow noise depending on its shape. In this study, numerical analysis was performed using ANSYS CFX ver. 18.1 to investigate the characteristics of flow noise and sound absorption. The thickness of the absorber shall be reduced or increased by $1 \mathrm{~mm}$ in relation to $86 \mathrm{~mm}$. In particular, if the absorber diameter increases by $2 \mathrm{~mm}$ in the newly designed model, SPL is shown at $121.55 \mathrm{~dB}$, down $9.3 \%$ from the reference model.
\end{abstract}

Keywords: Gaseous extinguishing nozzle, Absorbent, SPL (Sound pressure level), Flow noise, CFD (Computational fluid dynamics).

\section{Introduction}

Discharging the gaseous extinguishing agent generates a strong flow noise of about $139 \mathrm{~dB}$. This noise level makes the flow noise similar to fireworks because gas-based fire extinguishing systems use high pressure vessels of 100 bar or more for prompt fire suppression [1]. In particular, when the sound pressure level exceeds $130 \mathrm{~dB}$, the hard disk drive (HDD), which is an electronic product, is damaged and cannot be recovered. The vibrations generated by flow noise can cause head collisions in the magnetic layer of the disk surface. When a head crash occurs for the first time, the particle quickly collides and wears down in the storage chassis system $[2,3]$. Since the data storage device is damaged by the flow noise during the fire suppression process, the research is needed to decrease this noise. Jang [4] selected the nozzle hole configuration, the number of outlet holes and the arrangement of sound absorbents as design variables. And then reported that the experimentally selected optimal design parameter was reduced the noise generated from the porous air nozzles. Jeon et al. [5] investigated the discharge characteristics according to the discharging hole area in the gaseous extinguishing nozzle. In this study, the change of hole size, mass flow rate and injection pressure were set as design parameters. Meanwhile, the fire extinguishing agents from the nozzle can resolve the noise reduction by distributing the pressure through porous tubes. Lee [6] experimented with the flow noise of supersonic exhaust nozzles in porous tubes. The porosity of the porous tube caused both impact noise and turbulence mixture to occur. The noise reduction effect was an average of $5 \mathrm{~dB}$ as a result of changing the porosity of the tubes. Gwak et al. [7] studied the noise characteristics caused by supersonic jet airflow as the working fluid passed through the noise absorption material. By changing the shape of the absorbent, the pressure of the working fluid was reduced in the ceiling of the anechoic chamber. In addition, the intensity of the scratches produced by sound waves was clearly reduced at the nozzle outlets. Suh [8] selected diameter, length, and porosity of the absorbent body as design parameters. The loss of entry and noise characteristics resulting from passing noise absorption materials were then investigated according to the change in flow rate at the inlet. Experiments and numerical studies on absorber are currently being investigated, but most studies have been performed on packing density, porosity, and absorber arrangements. The sound pressure level was measured by establishing an observation point at a specified distance from the fire extinguisher, since noise reduction performance according to the form of absorbent shall be preceded. Following the numerical analysis, the performance improvement was tested using absorbent material of the silent fire extinguishing nozzle. The reference model for this study is the gaseous extinguishing nozzle provided by $\mathbf{J}$ company in Korea. The design parameters were selected as the ratio of the absorber thickness inside the nozzle to the diameter of the discharging hole.

\section{Numerical Analysis}

\subsection{Model Description}

Figure 1 shows a quarter of the gaseous extinguishing nozzle model. Gaseous extinguishing nozzle is largely divided into four 
Table 1: Design variables of the gaseous extinguishing nozzle.

\begin{tabular}{|c|c|c|c|}
\hline Case & $\begin{array}{l}\text { Discharging hole diameter, } \\
\mathrm{D}_{1}[\mathrm{~mm}]\end{array}$ & $\begin{array}{l}\text { Absorbent diameter, } \\
\mathrm{D}_{2}[\mathrm{~mm}]\end{array}$ & $\begin{array}{l}\text { Aspect ratio } \\
\left(D_{2} / D_{1}\right)\end{array}$ \\
\hline Case 1 & \multirow[t]{9}{*}{ 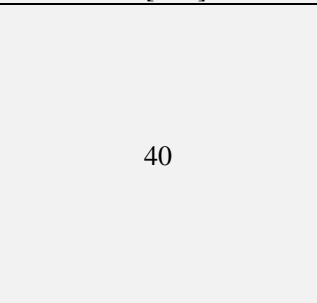 } & 82 & 2.050 \\
\hline Case 2 & & 83 & 2.075 \\
\hline Case 3 & & 84 & 2.100 \\
\hline Case 4 & & 85 & 2.125 \\
\hline Case 5 (Ref.) & & 86 & 2.150 \\
\hline Case 6 & & 87 & 2.175 \\
\hline Case 7 & & 88 & 2.200 \\
\hline Case 8 & & 89 & 2.225 \\
\hline Case 9 & & 90 & 2.250 \\
\hline
\end{tabular}

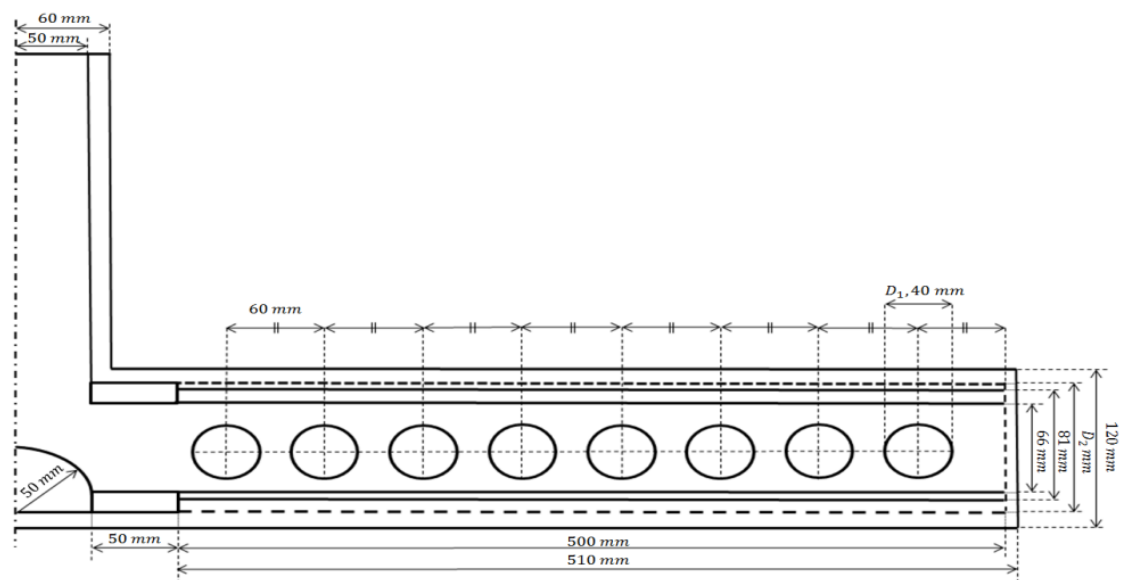

Fig. 1: A quarter configuration of the reference model of extinguishing nozzle.

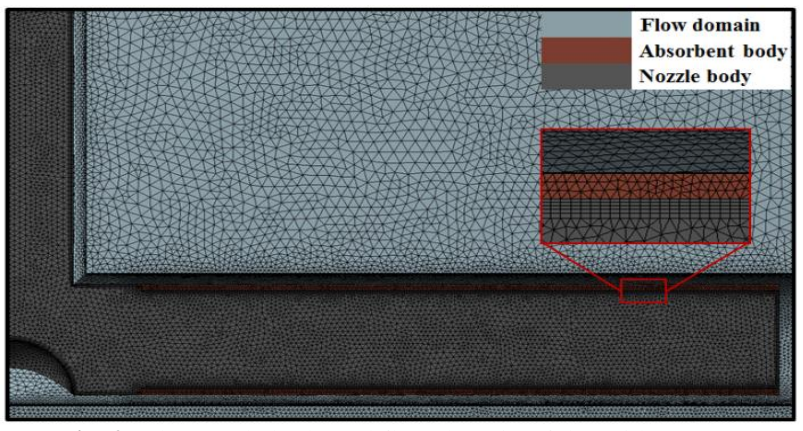

Fig. 2: The nozzle body and flow domain of the grid system.

parts as an inlet, the inner flow path, the absorbent area and the discharging hole. The number of discharging holes was 8 in a quarter of the nozzle, and the diameter was $40 \mathrm{~mm}$. The distance between the discharging holes was set to $20 \mathrm{~mm}$. Also, the flow area is composed of $620 \mathrm{~mm}$ in width, $100 \mathrm{~mm}$ in length, and $350 \mathrm{~mm}$ in height. The absorbent composed of aluminum was designed to abut the inner flow path in the shape attached to the inside of the nozzle, and the porosity was structured to 0.3 . The design parameter was selected as the diameter ratio of the discharging hole and the absorbent, and the diameter of the absorbent was changed based on thickness of $86 \mathrm{~mm}$. The nine different models were used to determine the performance of the design variables on the flow noise reduction of the extinguishing nozzle. Table 1 shows the cases according to design variables.

\subsection{Governing Equations}

The three-dimensional, unsteady, compressible, supersonic flow fields were calculated using the following governing equations:

\subsubsection{Continuity}

$\frac{\partial \rho}{\partial t}+\nabla \cdot(\rho u)=0$

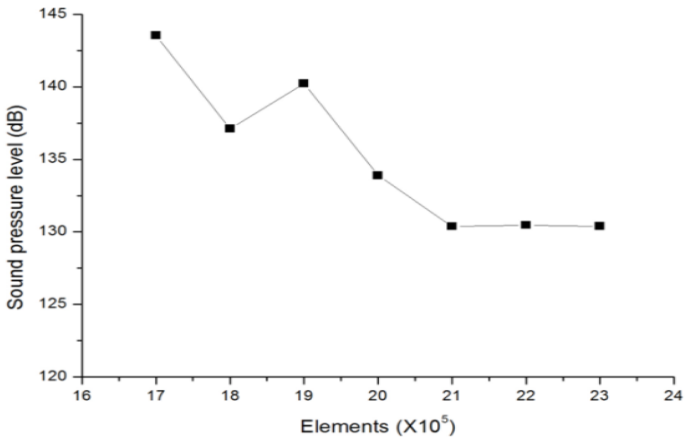

Fig. 3: The grid dependency test.

Momentum:

$\frac{\partial(\rho u)}{\partial t}+\nabla \cdot(\rho u u)=\nabla \cdot(\tau)-\nabla \cdot(p)$

where $\rho$ is the density of the working fluid, $u$ is the working fluid velocity, $p$ is the pressure, $\tau$ is the viscous stress tensor. In this study, numerical analysis was performed to investigate transient state and compressible flow fields using ANSYS CFX ver. 18.1. The transient analysis was calculated 250 time steps, which are divided by 0.02 seconds, under the condition that the working fluid was discharged for 5 seconds. The nitrogen $\left(\mathrm{N}_{2}\right)$ was used as the working fluid for the extinguishing agent, and the mass flow rate was set $1 \mathrm{~kg} / \mathrm{s}$ at the inlet of the nozzle. The SST (Shear Stress Transport) model proposed by Menter was used as a turbulence model to adequately simulate the flow separation phenomenon on the wall [9]. This turbulence model has the advantage of analyzing complex turbulence near the wall and accurately calculating the pressure-induced flow separation [10].

\subsection{Grid System}

Figure 2 shows a detailed grid system. Grid systems for analysis and absorbent systems are composed of tetrahedron mesh in the 
fluid domain. The overlap layers for calculation forms the five thicknesses to improve the precision of numerical analysis on the second. The ratio of the measured pressure to the reference pressure means the sound pressure level and can be calculated as follows [12]:

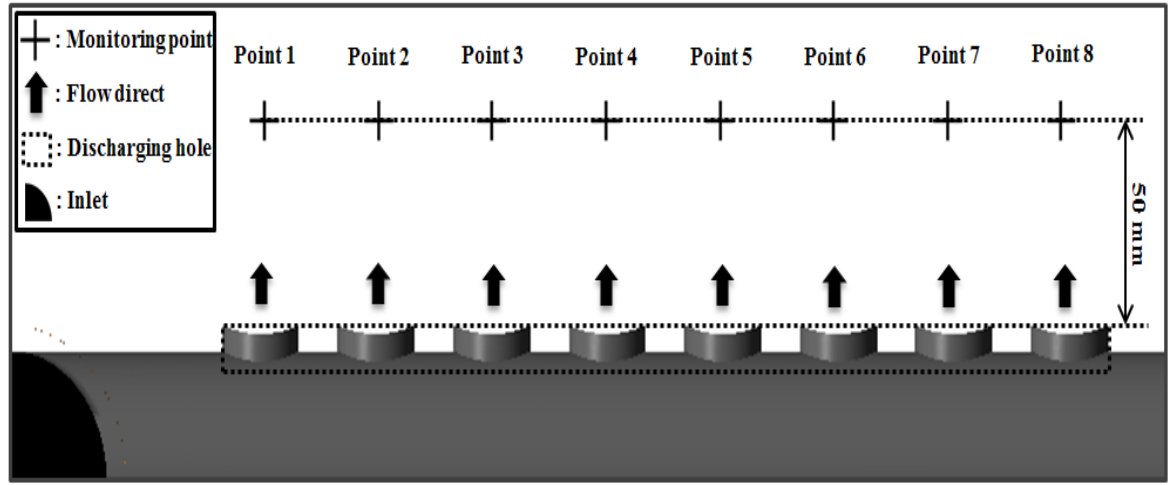

Fig. 4: The monitoring points of SPL in the vicinity of the discharging hole.

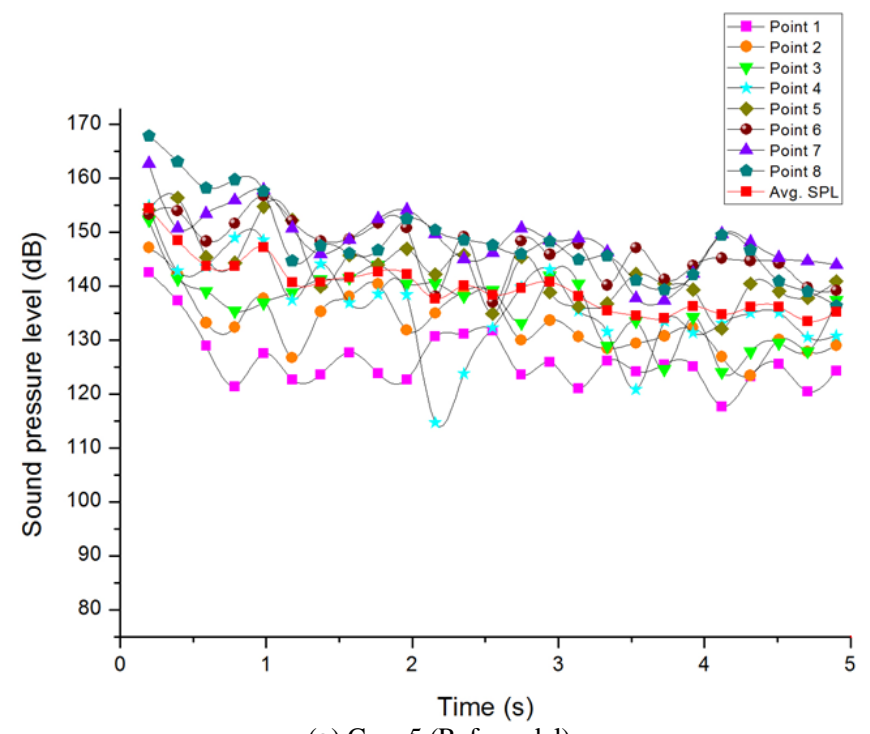

(a) Case 5 (Ref. model)

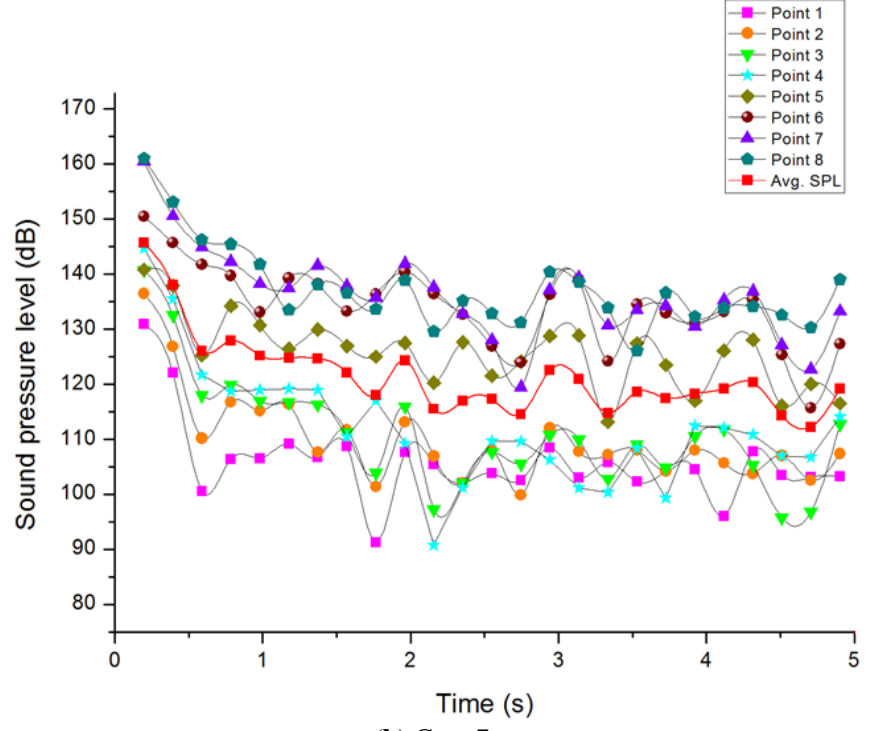

(b) Case 7

Fig. 5: Comparison results of SPL at 8 different points.

boundary layer. The first layer height was applied under the condition of $\mathrm{y}^{+}<75$ from the wall. Furthermore, a grid dependency test was performed as shown in Fig. 3. The lowest sound pressure levels were investigated as averages, increasing each of the 100,000 grids to 2.3 million from the 1.7 million basic values Average sound pressure values, which are performance indicators, tend to eventually converge at $130.85 \mathrm{~dB}$ when the number of mesh is more than 2.1 million.

\section{Results and Discussion}

\subsection{Noise Level}

Sound pressure levels are derived from three-dimensional transient RANS analysis [11]. And the flow noise was observed at eight monitoring points as shown in Fig. 4. Each monitoring point is measured at a point $50 \mathrm{~mm}$ vertically away from the discharge hole and the pressure was recorded every 0.02 seconds to calculate SPL. Sound pressure indicates the volume of sound produced when the sound waves are transmitted by vibrations in the air layer. At a speed of approximately $334 \mathrm{~m} / \mathrm{s}$ the volume of sound can be expressed as quantitative sound energy in the standard atmosphere and applied a force of $1 \mathrm{~m}^{2}$ in a vertical direction for 1
$S P L=20 \log \frac{p}{p_{0}}$

where SPL is the sound pressure level, $p_{0}$ is the reference sound pressure in the atmosphere, and $p$ is the sound pressure measured at the monitoring point. By testing the grid dependence, the average SPL result for the reference model was previously calculated at $130.85 \mathrm{~dB}$ with an absorbent of $86 \mathrm{~mm}$. The mean SPL with $121.55 \mathrm{~dB}$ was observed in Case 7 of the variation in absorption. This is the lowest level of noise among the nine cases. A reduction in flow noise was identified by changing the shape of the absorber. As a result, in Case 7, the average flow noise was reduced by 9.3\%. The results are shown in Fig. 5 .

\subsection{Vortex Structure}

The vortex structure inside and outside the nozzle is shown in Fig. 6. It can be graphically represented within the nozzle by vortex forming and turbulence. In this study, the vortex was investigated at 5 second points during the total discharge time. Case 5 is a reference model that has a significantly greater vortex structure than any other case. In particular, it can be seen that the formation of vortex is noticeably structured at the end area of the nozzle. This is corresponds to the highest noise level from points 6 to 8 . Thus, the vortex core of Case 7 produces a much lower flow noise than Case 5. As a result of the vortex structure, it can be seen that the 
turbulence inside the nozzle affects the flow noise. To understand this trend, the agent flowing through the inlet is represented by the

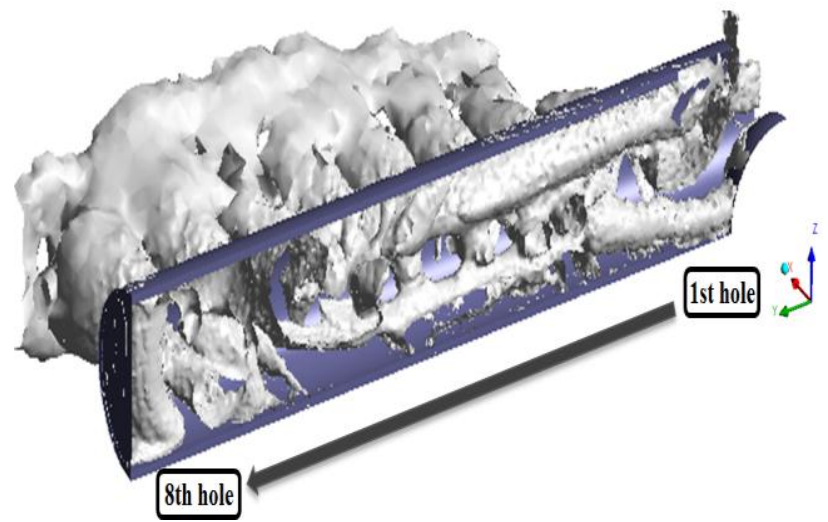

(a) Case 5 (Ref.)

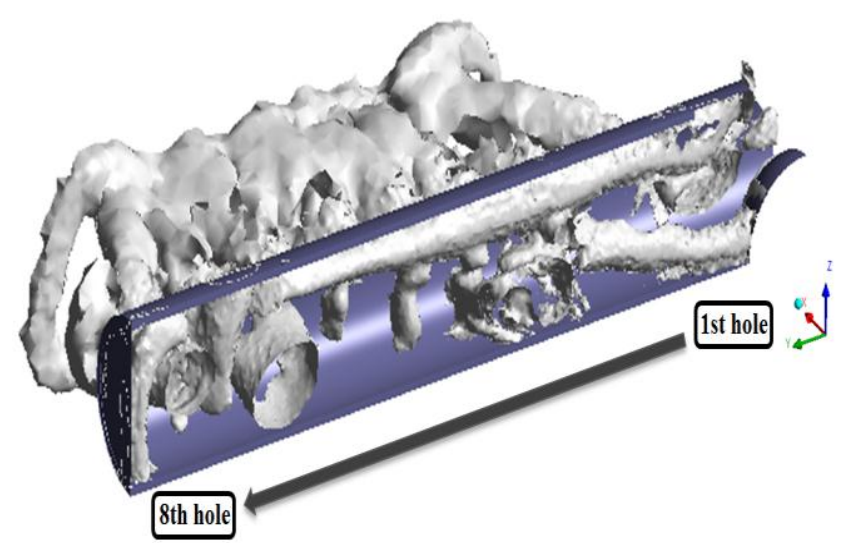

(b) Case 7

Fig. 6: Comparison of vortex structure between reference and improved model at $5 \mathrm{~s}$.

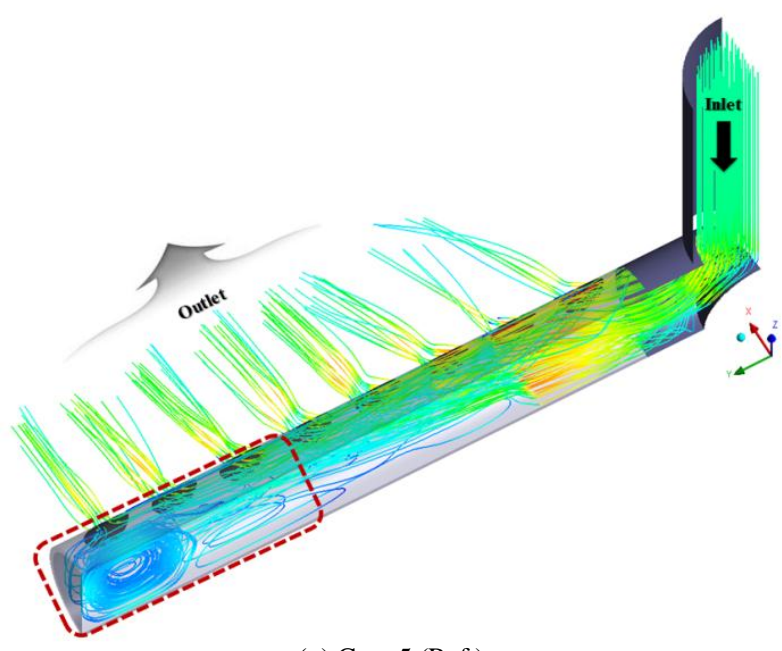

(a) Case 5 (Ref.)

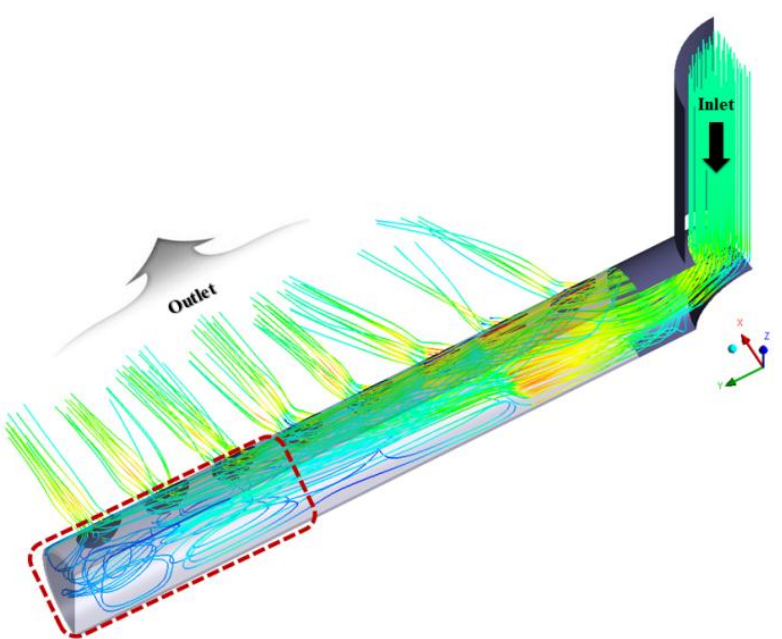

(b) Case 7

Fig. 7: Vortex structure visualized by $\mathrm{N}_{2}$ velocity and streamlines.

Velocity component of Fig. 7. As shown by the red box, the area near No. 8 was composed of relatively dense vortices as compared to No. 6 and 7. In case of swirling strength, the average swirling strength of the reference model is $10054.54 \mathrm{~s}^{-1}$, and the improved model Case 7 is $949 \mathrm{~s}^{-1}$ in the vortex structure. This is similar to the tendency of SPL results. Based on the results, vortex structure is measured as a factor affecting flow noise.

\section{Conclusions}

In this study, the effect of changes in the shape of absorbent on the flow noise of gas fire nozzles was investigated as a numerical method. To reduce the flow noise, aluminum absorption areas are attached inside the nozzle. The numerical analysis was then performed by setting the aspect ratio of the absorber and the discharge hole as a design variable. The results of this study are summarized as follows:

1) The thickness of the absorbent in the nozzle affects the flow noise and vortex structure.

2) The flow noise of the release agent increases as the vortex is generated near exits No. $6 \sim 8$ of the internal flow path.

3) If the absorbent diameter increases by $2 \mathrm{~mm}$ in the newly designed model, SPL is shown at $121.55 \mathrm{~dB}$, down $9.3 \%$ from the reference model.

\section{Acknowledgement}

This research was supported by a grant (18TBIP-C127226-02) from commercialization of national transportation technology funded by the Ministry of Land, Infrastructure and Transport of the Korean Government.

\section{References}

[1] Siemens, "Intelligent Extinguishing Solutions from Siemens. Sinorix CDT-Innovative, Sustainable, and Safe," https://www.siemens.com/download?A6V10082135, 2011.

[2] Siemens, "Silent Extinguishing Disruptions to hard disk drives caused by inert gas extinguishing systems-analysis and measures for the safe operation of storage systems," https://www.siemens.com/download?A6V10699087, 2015.

[3] Dutta, Trinoy and A. R. Barnard, "Performance of Hard Disk Drives in High Noise Environments," Noise Control Engineering, vol. 65, no. 5, pp: 386-395, 2017.

[4] S. W. Jang, "An Experimental Study on Noise Characteristics of Multi Hole Air Nozzle," Ph.D. diss., Seoul National University of Science and Technology, Korea, 2003 (in Korean).

[5] H. K. Jeon, Y. S. Choi and J. T. Park, "The Effects of the Area of Openings on the Performance of a CO2 Extinguishing System - The CFD Simulations of the Oil Surface Fire in a Machine Room," Fire Science and Engineering, vol. 22, no. 1, pp: 1-9, 2008 (in Korean).

[6] D. H. Lee, "A Study on the Aerodynamic Noise of a Supersonic Exhaust Nozzle of Perforated Tube," Transactions of the Korean 
Society for Noise and Vibration Engineering, vol. 9, no. 1, pp: 113120, 1999 (in Korean).

[7] Y. H. Kweon, M. Tsuchida, Y. Miyazato, T. Aoki, H. D. Kim, T. Setoguchi, "The effect of reflector with sound-absorbing material on supersonic jet noise," Journal of Thermal Science, vol. 14, no. 1, pp. 22-27, 2005.

[8] G. W. Suh, "A Study on the Acoustic Characteristics of Jet Noise from Orifices and the Performance of Diffuser Silencer," Ph.D. diss., University of Soongsil, Korea, 1993 (in Korean).

[9] F. R. Menter, "Two-Equation Eddy-Viscosity Turbulence Models for Engineering Applications," American Institute of Aeronautics and Astronautics, vol. 32, no. 8, pp: 1598-1605, 1994.

[10] F. R. Menter, M. Kuntz and R. Langtry, "Ten Years of Industrial Experience with the SST Turbulence Model," Proceeding of the 4th International Symposium on Turbulence, vol. 1, pp: 632-652, 2003.

[11] P. Arun kumar, P. Senthil and K. Naveen kumar, "Analysing Mass Flow Rate and Noise Level of Blowers through Ansys CFX Solver," Australian Journal of Mechanical Engineering, vol. 15, no. 3, pp: 175-181, 2017.

[12] M. P. Norton, D. G. Karczub, "Fundamentals of Noise and Vibration Analysis for Engineers," Cambridge, vol. 10, no. 2, pp: 282 $289,2003$. 